\title{
General Derivation of the Sets of Pedigrees with the Same Kinship Coefficients
}

\author{
Nádia Pinto ${ }^{a-c}$ Pedro V. Silva ${ }^{b, c}$ António Amorim ${ }^{a, b}$ \\ a Instituto de Patologia e Imunologia Molecular da Universidade do Porto (IPATIMUP), b Faculdade de Ciências da \\ Universidade do Porto, and ${ }^{\complement}$ Centro de Matemática da Universidade do Porto, Porto, Portugal
}

\section{Key Words}

Identity-by-descent - Extended pedigrees - Distinguishing pedigrees $\cdot$ Genotypic probabilities $\cdot$ Kinship testing

\begin{abstract}
Quantification of kinships between two individuals using unlinked autosomal markers rests upon the identity-bydescent (IBD) probabilities among their four alleles at a locus because they determine the algebraic expressions of the joint genotypic probabilities. Nevertheless, some pedigrees share the same IBD probabilities and are therefore indistinguishable using those markers. Examples of these pedigrees were previously described, such as the case of half-siblings, grandparent-grandchild and avuncular, but a general analysis has not been attempted. The aim of this study is to present a systematic and mathematically supported framework where considering unlinked autosomal markers complete sets of indistinguishable pedigrees linking two non-inbred individuals are generally derived. In our work, complete sets of pedigrees with the same IBD partitions are formally established and mathematically treated, considering kinships linking any pair of non-inbred individuals, whether they are related just maternally or paternally, or both. Moreover, general expressions for IBD partitions, and consequently for joint genotypic probabilities, are derived considering a simple counting rule based on two 'atom' pedigrees: parent-
\end{abstract}

\section{KARGER}

Fax +4161306 1234 E-Mail karger@karger.ch www.karger.com
(C) 2010 S. Karger AG, Basel 0001-5652/10/0703-0194\$26.00/0

Accessible online at: www.karger.com/hhe child and full-siblings. Besides the theoretical formalization of the problem, the developed framework has potential applications in forensics as well as in breeding strategies design and in conservation studies.

Copyright $\odot 2010$ S. Karger AG, Basel

\section{Introduction}

The study of genetic relationships, involving genealogical estimation and pedigree reconstruction, has been a matter of great theoretical and practical interest not only in humans but also in animal and plant populations, for example in breeding and conservation studies.

In humans, the estimation of genetic relatedness is relevant in fields such as genetic mapping via linkage and association studies. Considering unlinked markers, several applications emerge, for example, in the forensic field [1] associated with standard problems such as immigration cases [2], paternity testing [3] or identification problems in mass disasters [4]. Several approaches for estimation of genetic relatedness were developed considering independent markers [5-7].

The motivation for this work was triggered by the fact that it was shown [8] that some pedigrees share the same (autosomal) kinship coefficients and are thus indistinguishable when using this type of unlinked markers. The 
most commonly cited example is the set of pedigrees grandparent-grandchild, avuncular and half-siblings, involving a pair of non-inbred individuals.

Indeed, in some circumstances, the referred theoretical feature of some pedigrees can constitute a major limitation for genetic analysis and conclusions. A typical example is shown in the following forensic case in which kinship testing is required in the context of an inheritance claim.

Example: A woman W claims to be a daughter of a rich man $\mathrm{M}$ who recently passed away, aiming at part of his inheritance. The only available relative of $\mathrm{M}$ for genetic comparison is male $S$, one of the two recognized sons of $\mathrm{M}$. However, $\mathrm{S}$ states that $\mathrm{W}$ could be the daughter of his unavailable brother $\mathrm{B}$, instead of his father $\mathrm{M}$, and wants to compare both possibilities. Thus, genetic experts have to compare the pedigrees depicted in figure 1 .

Without any other type of information, it is known that the possibilities under consideration are genetically indistinguishable when considering the standard forensic practice of using unlinked autosomal markers.

However, it must be said that the referred hypotheses are, at least theoretically, differentiable when considering other types of markers. An early discussion on linked autosomal markers is provided by Thompson [8]. Thompson and Meagher revisited and extended the discussion on this issue [9], and it is additionally debated by Egeland and Sheehan [10]. A theoretical framework for X-chromosomal markers is given by Pinto et al. [11].

Our goal is to present a systematic and mathematically supported framework where complete sets of indistinguishable pedigrees linking two non-inbred individuals are generally derived, considering unlinked autosomal markers.

The theoretical study of genetic relationships is always linked with the concept of identity-by-descent. Two alleles are said to be identical-by-descent (or IBD) if they descend from the same ancestral allele. Moreover, two individuals are said to be related if their coancestry coefficient is nonnull, or in other words if an allele of one individual can be IBD to an allele of the other individual [12].

Considering two individuals, the partitions of the identity-by-descent among their four alleles are well established since at least 1970 [12], and each IBD coefficient is known as a Jacquard's coefficient [5]. For non-inbred individuals, the nine IBD partitions can be reduced to three: $k_{2}$ - probability of sharing two IBD alleles, $k_{1}$ - probability of sharing exactly one IBD allele, and $k_{0}$ - probability of sharing none [see 5 for a comprehensive review]. Consequently, there are only three extreme cases of pedigrees

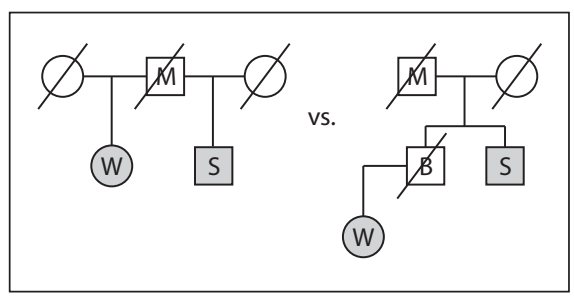

Fig. 1. Pedigree paternal half-brother-sister versus pedigree paternal uncle-niece. Only individuals in grey are available for genetic testing.

having one and only one non-null IBD partition (probability equals 1): identical twins or identity (for which $k_{2}=$ 1 , and $k_{1}=k_{0}=0$ ), parent-child (for which $k_{1}=1$, and $k_{2}=$ $k_{0}=0$ ) and unrelated (for which $k_{0}=1$, and $k_{2}=k_{1}=0$ ).

Thus, characterizing the relationship between two individuals rests upon the respective IBD probabilities because their joint genotypic probabilities depend solely on these parameters and on allele frequencies [5].

It is well known since at least 1975 [8] that there are pedigrees with the same IBD partitions and, consequently, with the same associated joint genotypic probabilities. Thus, pedigrees with the same IBD partitions are said to belong to the same autosomal kinship class because they are indistinguishable by the use of unlinked autosomal markers [for a discussion on this topic see 13].

In this paper, considering the autosomal mode of transmission and two non-inbred individuals, a theoretical framework for identity-by-descent is established, aiming at a description of all pedigrees belonging to each autosomal kinship class. Moreover, since IBD partitions are easily derived, algebraic expressions for the joint genotypic probabilities are also presented. Indeed, the IBD partitions, even for extended pedigrees, are obtained just by considering the minimal number of extra individuals beyond the 'atom' pedigrees (parent-child and full-siblings) that have to be considered in each case.

With respect to the framework we are presenting, in the section 'Conclusions' we generalize the most complete table, by far, that we have found in the literature [table 2 in reference 8], in which IBD probabilities are reported for some genealogical relationships (in absence of inbreeding). Indeed, in table 9 we present all the pedigrees belonging to the autosomal kinship classes mentioned in the existing table [8], undertaking a general and formal analysis of the genealogical relationships regardless of the probability of such pedigrees emerging in the population.

In spite of our study being limited to unlinked and autosomal markers and non-inbred individuals, it must be 
Table 1. 'Atom' pedigrees: parent-child and full-siblings, involving non-inbred individuals and respective IBD partitions

\begin{tabular}{lll}
\hline & Parent-child & Full-siblings \\
\hline Pedigrees & & \\
& & \\
IBD partitions & $k_{0}=0$ & $k_{0}=1 / 4$ \\
& $k_{1}=1$ & $k_{1}=1 / 2$ \\
& $k_{2}=0$ & $k_{2}=1 / 4$ \\
\hline
\end{tabular}

Table 2. Pedigrees resulting from adding one extra branch to the atom pedigrees (parent-child and full-siblings) and respective IBD partitions

\begin{tabular}{lll}
\hline & Half-siblings & $\begin{array}{l}\text { Grandparent- } \\
\text { grandchild }\end{array}$ \\
\hline Pedigrees & Avuncular \\
IBD & $k_{0}=1 / 2$ & \\
partitions & $k_{1}=1 / 2$ \\
& $k_{2}=0$ & \\
\hline
\end{tabular}

remarked that the existence of indistinguishable pedigrees is verified with regard to inbred individuals [see e.g. 11] and other types of markers, for instance, heterosomal [11] and autosomal linked markers [14, 15].

\section{Definitions}

In this paper, only the unlinked autosomal mode of transmission and pedigrees involving two non-inbred individuals will be analyzed. Moreover, we assume that alleles are codominant and that mutation is absent.

The framework for the analysis of any genetic relationship involving two non-inbred individuals (identical twins and unrelated individuals excepted) depends on the definition of the 'atom' pedigrees: parent-child and full-siblings, whose IBD partitions are well known and established (table 1).

Indeed, any pedigree involving two non-inbred individuals can be formally described considering these two 'atom' pedigrees and the minimal number of extra indi- viduals that is necessary to be considered. In the following, we will designate as 'extra branch' or 'extra individual' each generation or individual that is necessary to join to one of the two 'atom' pedigrees for defining the relationship between the individuals. For example, in each of the pedigrees half-siblings, grandparent-grandchild and avuncular, it is necessary to add one extra individual to one 'atom' pedigree (to the parent-child pedigree in the first two cases and to the full-siblings for the third case; table 2).

A pedigree involving two individuals is said to be uniparental if they are related by exactly one route: maternal or paternal. Assuming that individuals are related by just one route is equivalent to assuming that the probability of the individuals sharing two IBD alleles $k_{2}$ is null. Note that $k_{2} \neq 0$ implies that the individuals could have received two IBD alleles from both maternal and paternal origin.

On the other hand, when two individuals are related both maternally and paternally by exactly two pedigrees, besides full-siblings, they are said to be related through a biparental pedigree, for which $k_{2}$ is non-null. The most common example is the case of double first cousins, but many others involving non-inbred individuals are possible. In table 3 some examples are depicted.

In order to formally establish biparental pedigrees we have to decompose those pedigrees in the two associated uniparental pedigrees, considering the respective number of extra individuals.

\section{Uniparental Pedigrees}

Besides parent-child pedigrees, we call pedigrees that relate two individuals by exactly just one path (maternal or paternal) uniparental pedigrees, for which $k_{2}=0$.

As stated before, the simplest example that can be used to construct pedigrees joining individuals to the atom pedigrees is when exactly one extra individual is considered (and the pedigrees half-siblings, avuncular and grandparent-grandchild are obtained; table 2). In table 4, uniparental pedigrees and respective IBD partitions considering 1, 2 or 3 extra branches beyond 'atom' pedigrees are depicted.

In table 4, we can observe that pedigrees with the same number of extra branches belong to the same autosomal class since they have the same IBD partitions. Indeed, this can be extended to all possible numbers of extra individuals that we can join to one of the two 'atom' pedigrees. The uniparental pedigrees, for which it is necessary to consider a minimal number $n$ of extra individuals, have IBD partitions that depend only on the number $n$. Con- 
Table 3. Examples of biparental pedigrees

Biparental pedigrees
Half-siblings
whose non-
identical
parents are
parent-child
Half-siblings
whose non-
identical
parents are
full-siblings
Double first
cousins
Half-siblings
whose non-
identical parents
are half-siblings
Double half-avuncular

sidering a uniparental pedigree, it is necessary to consider $n$ extra branches:

$$
k_{0}^{(n)}=1-(1 / 2)^{n}, k_{1}^{(n)}=(1 / 2)^{n}, k_{2}^{(n)}=0,
$$

where $k_{i}^{(n)}, i=0,1,2$, is the $i$-th IBD partition of a uniparental pedigree with $n$ extra branches. For a mathematical proof see Appendix I.

Therefore, we can say that two uniparental pedigrees involving two non-inbred individuals belong to the same autosomal kinship class if and only if they have the same number of extra individuals.

Moreover, it is also proved that in the autosomal kinship class where uniparental pedigrees have $n$ extra individuals, there are $n+2$ different pedigrees. In other words, we can state that there are $n+2$ indistinguishable pedigrees by the use of unlinked autosomal markers, when $n$ extra individuals are considered. For examples, see table 4

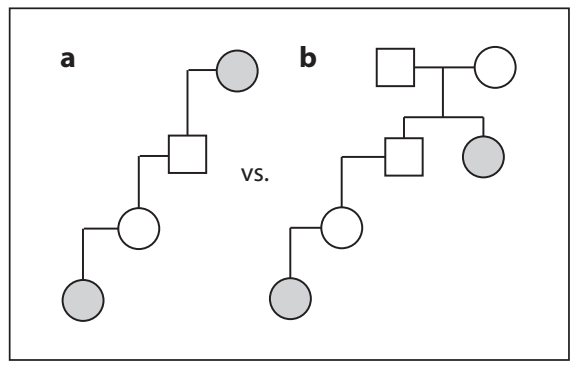

Fig. 2. Possibilities at stake considering the fictional example described in the text (available samples represented in grey).

where the 3, 4 and 5 pedigrees which can be constructed considering 1, 2 and 3 extra individuals, respectively, are presented. For a mathematical proof, see Appendix II.

Concerning uniparental pedigrees, in table 5 we present the IBD partitions as well as the number of pedigrees belonging to the same autosomal kinship class depending on the number $\mathrm{n}$ of extra individuals in the pedigrees.

Admittedly, inspired by the famous case example of the Russian Romanov dynasty and the controversy surrounding the identity of Anna Anderson Manahan who claimed, in 1920, to be Duchess Anastasia, daughter of Tsar Nicholas II [16], we present a fictional example, again in the forensic field, where the use of unlinked markers would be useless. We show how the method we present can be useful to easily discard the genetic analysis considering that mode of transmission. Indeed, the example was chosen in order to evidence the simplicity of the method when applied to a pair of pedigrees whose IBD partitions we did not find described in the literature.

Example: In the past, three members of the same family, father, mother and one daughter, disappeared in a small village without trace, which caused some people to suspect that they were the victims of a hideous crime. The couple had another son who did not disappear. The surviving son himself had one daughter and later one granddaughter. Time passed, and the only descendent alive of the missing couple is now this great-granddaughter.

Recently, some lumberjacks found a human skeleton in a wood near the home of the disappeared family. Genetic expertise determined that these were a woman's bones, raising the suspicion that it belonged either to the missing mother or daughter.

Assuming that those are the only hypotheses at stake and taking into account that the only comparative sample available is the one of the great-granddaughter, in order to compare both possibilities, we have to consider the pedigrees depicted in figure 2 . 
Table 4. Pedigrees and respective IBD partitions considering zero, one, two or three extra branches beyond the 'atom' pedigrees: parent-child and full-siblings

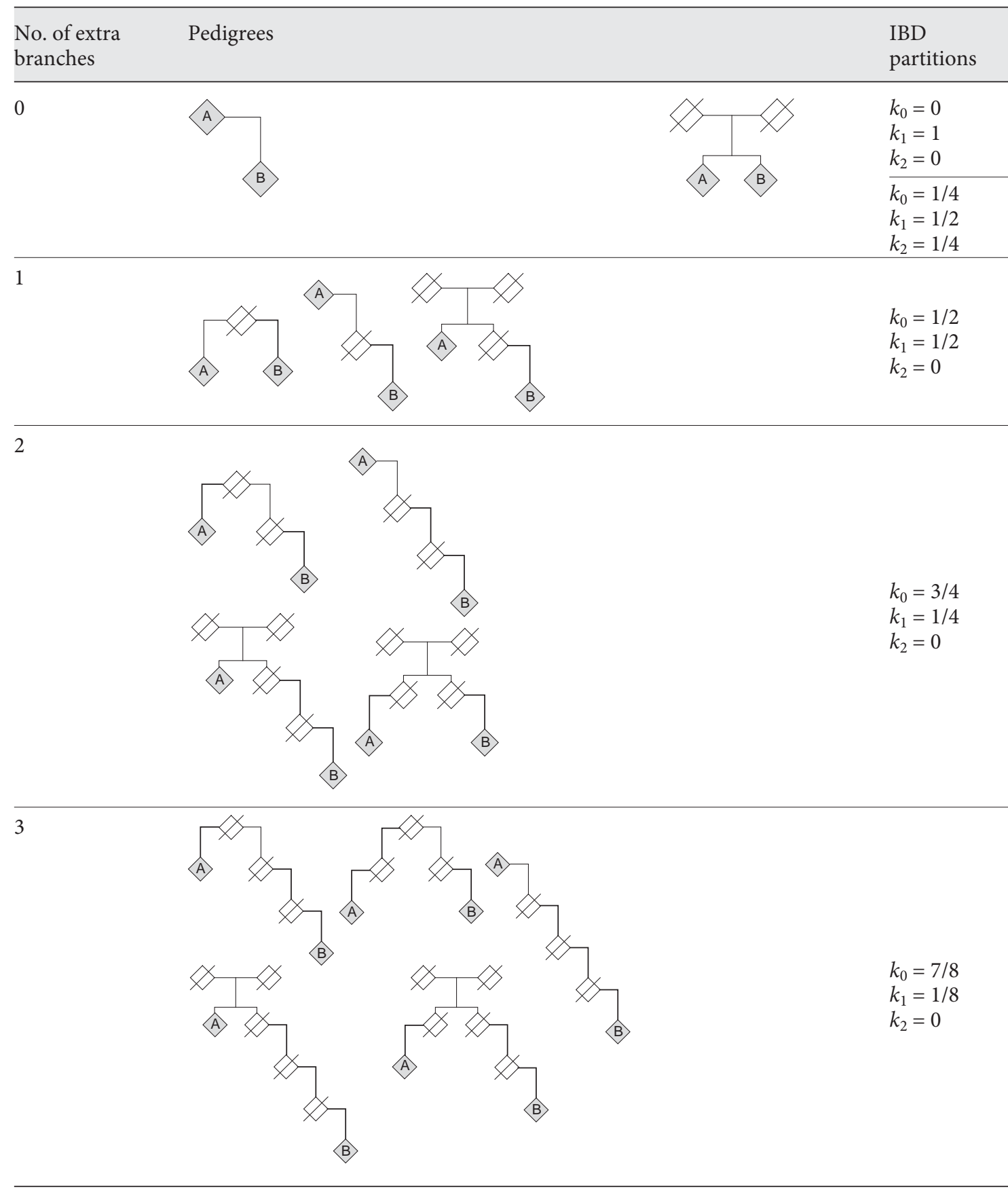

Apart from the practical limitations related to the lack of power of unlinked markers when analyzing extended (more than second-degree) pedigrees [17], the use of unlinked autosomal markers is useless because the depicted pedigrees have the same IBD partitions and are, therefore, indistinguishable with regard to this mode of trans- mission. Indeed, according to the counting rule presented, it can be easily seen that in both pedigrees, there are exactly two extra individuals beyond the respective 'atom' pedigree (parent-child in pedigree $2 \mathrm{a}$ and full-siblings in pedigree $2 b)$. 


\section{Biparental Pedigrees}

As stated earlier, except for the atom pedigree full-siblings, we call biparental pedigrees those where the individuals are related by exactly two pedigrees: one via paternal and the other via maternal pedigrees. In such cases, the IBD partition $k_{2}$ is non-null, since individuals can have two IBD alleles: each one from paternal and maternal inheritance. In table 3, we present some examples of this type of pedigrees.

It is easily seen that one biparental pedigree is a composite of two uniparental pedigrees. In table 6, we present the decomposition of the biparental pedigrees depicted in table 3 into two uniparental pedigrees as well as the number of extra individuals considered in each pedigree.

To perform the IBD partitions for biparental pedigrees $\mathrm{BP}$, we need to consider the IBD partitions associated with each of the respective uniparental pedigrees $\left(\mathrm{P}_{1}\right.$ and $\mathrm{P}_{2}$ ), since by definition

$$
\begin{aligned}
& k_{0}(\mathrm{BP})=k_{0}\left(\mathrm{P}_{1}\right) \cdot k_{0}\left(\mathrm{P}_{2}\right) \\
& k_{1}(\mathrm{BP})=k_{0}\left(\mathrm{P}_{1}\right) \cdot k_{1}\left(\mathrm{P}_{2}\right)+k_{1}\left(\mathrm{P}_{1}\right) \cdot k_{0}\left(\mathrm{P}_{2}\right) \\
& k_{2}(\mathrm{BP})=k_{1}\left(\mathrm{P}_{1}\right) \cdot k_{1}\left(\mathrm{P}_{2}\right)
\end{aligned}
$$

where $k_{i}(\mathrm{R}), i=0,1,2$, is the $i$-th IBD partition associated with relationship $\mathrm{R}$.

Indeed, the presented expressions are obtained by definition since, considering that individuals are non-inbred and for each individual the maternal relationship is independent from the paternal, the IBD partitions associated with the biparental pedigree only depend on the IBD partitions associated with the respective uniparental pedigrees. The IBD partition $k_{0}$ is the probability of the individuals not sharing any IBD allele; therefore, this probability is equal to the product of the probabilities of individuals not sharing any IBD allele from neither the maternal nor paternal path. On the other hand, $k_{2}$ is the probability of the individuals sharing two IBD alleles and, therefore, we have to consider the possibility of the individuals having received IBD alleles both paternally and maternally. Finally, to determine the probability of the individuals sharing exactly one IBD allele, we have to consider the possibility of the individuals sharing one IBD allele from the maternal path and none by the paternal route, and vice versa.

Thus, as in order to describe uniparental pedigrees $P_{1}$ and $\mathrm{P}_{2}$ we have to consider $n_{1}$ and $n_{2}$ extra individuals, respectively, using the expressions presented in the section Uniparental Pedigrees for IBD partitions of those types of pedigrees, for a biparental pedigree $\mathrm{BP}$

Sets of Pedigrees with the Same Kinship Coefficients
Table 5. IBD partitions of the uniparental pedigrees for which it is necessary to consider extra branches at the 'atom' pedigrees

\begin{tabular}{llll}
\hline $\begin{array}{l}\text { No. of extra } \\
\text { branches }\end{array}$ & $\begin{array}{l}\text { No. of uni- } \\
\text { parental pedigrees }\end{array}$ & $\begin{array}{l}\text { IBD partitions of each } \\
\text { uniparental pedigree }\end{array}$ \\
\cline { 3 - 4 } & & $k_{0}$ & $k_{1}$ \\
\hline 1 & 3 & $1 / 2$ & $1 / 2$ \\
2 & 4 & $3 / 4$ & $1 / 4$ \\
3 & 5 & $7 / 8$ & $1 / 8$ \\
$\ldots$ & $\ldots$ & $\ldots$ & $\ldots$ \\
$n$ & $n+2$ & $1-(1 / 2)^{n}$ & $(1 / 2)^{n}$ \\
\hline
\end{tabular}

Note that for uniparental pedigrees $k_{2}$ is null.

$$
\begin{aligned}
& k_{0}=1-(1 / 2)^{n_{1}}-(1 / 2)^{n_{2}}+(1 / 2)^{n_{1}+n_{2}}, \\
& k_{1}=(1 / 2)^{n_{1}}+(1 / 2)^{n_{2}}-(1 / 2)^{n_{1}+n_{2}-1} \text { and } \\
& k_{2}=(1 / 2)^{n_{1}+n_{2}} .
\end{aligned}
$$

Moreover, it can be proved that two biparental pedigrees belong to the same autosomal kinship class if and only if their uniparental associated pedigrees both have the same number of extra individuals.

For a mathematical proof, see Appendix III, and for examples, see table 7, where the IBD partitions are presented for the biparental pedigrees described in table 6 . In table 7, it should be noted that in spite of the pedigrees 'double first cousins' and 'half-siblings whose non-identical parents are half-siblings', for example, having the same total number of extra individuals, they belong to different autosomal kinship classes since the respective uniparental pedigrees contain a different number of extra individuals.

Furthermore, each autosomal kinship class involving biparental pedigrees has a finite number of different pedigrees, depending exclusively on the number of extra individuals $n_{1}$ and $n_{2}$ considered in each uniparental pedigree associated, as previously defined.

The study of uniparental pedigrees has shown that considering $n$ extra individuals, there are $n+2$ pedigrees belonging to the same autosomal kinship class (see Uniparental Pedigrees). However, considering biparental pedigrees involving individuals related both maternally and paternally but non-inbred, we have to discard uniparental pedigrees where both parents of one individual are ancestors of the other by the same path. This situation happens in two circumstances: when the $n$ extra individuals are considered successively linked to the child in the atom pedigree parent-child or successively linked to one of the

Hum Hered 2010;70:194-204 
Table 6. Biparental pedigrees (BP) depicted in table 3, presented as a composite of two uniparental pedigrees $\mathrm{P}_{1}$ and $\mathrm{P}_{2}$

\begin{tabular}{|c|c|c|c|c|c|}
\hline \multirow{2}{*}{\multicolumn{2}{|c|}{$\begin{array}{l}\text { Biparental pedigrees } \\
\mathrm{BP}=\mathrm{P}_{1}+\mathrm{P}_{2}\end{array}$}} & \multicolumn{4}{|c|}{ Uniparental pedigrees } \\
\hline & & \multicolumn{2}{|l|}{$\mathrm{P}_{1}$} & \multicolumn{2}{|l|}{$\mathrm{P}_{2}$} \\
\hline description & $n$ & description & $n_{1}$ & description & $n_{2}$ \\
\hline Half-siblings whose non-identical parents are parent-child & 3 & half-siblings & 1 & half-avuncular & 2 \\
\hline Half-siblings whose non-identical parents are full-siblings & 3 & half-siblings & 1 & first cousins & 2 \\
\hline Double first cousins & 4 & first cousins & 2 & first cousins & 2 \\
\hline Double half-avuncular & 4 & half-avuncular & 2 & half-avuncular & 2 \\
\hline Half-siblings whose non-identical parents are half-siblings & 4 & half-siblings & 1 & half-first cousins & 3 \\
\hline
\end{tabular}

The number of extra individuals $n_{1}$ and $n_{2}$, considered for each uniparental pedigree $\mathrm{P}_{1}$ and $\mathrm{P}_{2}$, respectively, are also presented as well as the total number $n$ of extra individuals $n=n_{1}+n_{2}$ considered for each biparental pedigree (which is associated, by definition, with two uniparental pedigrees).

Table 7. IBD partitions of the biparental pedigrees (BP) described in table 6 (and depicted in table 3 )

\begin{tabular}{|c|c|c|c|c|c|c|}
\hline \multirow{2}{*}{\multicolumn{2}{|c|}{$\begin{array}{l}\text { Biparental pedigrees } \\
\mathrm{BP}=\mathrm{P}_{1}+\mathrm{P}_{2}\end{array}$}} & \multicolumn{4}{|c|}{ Uniparental pedigrees } & \multirow{3}{*}{$\begin{array}{l}\text { IBD } \\
\text { partitions }\end{array}$} \\
\hline & & \multicolumn{2}{|l|}{$\mathrm{P}_{1}$} & \multicolumn{2}{|l|}{$\mathrm{P}_{2}$} & \\
\hline description & $n$ & description & $n_{1}$ & description & $n_{2}$ & \\
\hline Half-siblings whose non-identical parents are parent-child & 3 & half-siblings & 1 & half-avuncular & 2 & \multirow{2}{*}{$\begin{array}{l}k_{0}=3 / 8 \\
k_{1}=1 / 2 \\
k_{2}=1 / 8\end{array}$} \\
\hline Half-siblings whose non-identical parents are full-siblings & 3 & half-siblings & 1 & first cousins & 2 & \\
\hline Double first cousins & 4 & first cousins & 2 & first cousins & 2 & \multirow{2}{*}{$\begin{array}{l}k_{0}=9 / 16 \\
k_{1}=3 / 8 \\
k_{2}=1 / 16\end{array}$} \\
\hline Double half-avuncular & 4 & half-avuncular & 2 & half-avuncular & 2 & \\
\hline Half-siblings whose non-identical parents are half-siblings & 4 & half-siblings & 1 & half-first cousins & 3 & $\begin{array}{l}k_{0}=7 / 16 \\
k_{1}=1 / 2 \\
k_{2}=1 / 16\end{array}$ \\
\hline
\end{tabular}

It should be noted that in spite of the pedigrees 'double first cousins' and 'half-siblings whose non-identical parents are half-siblings' for example having the same total number of extra individuals, they belong to different autosomal kinship classes since the respective uniparental pedigrees $\mathrm{P}_{1}$ and $\mathrm{P}_{2}$ contain a different number of extra individuals.

two full-siblings in the atom pedigree full-siblings. Both described situations correspond to pedigrees where, using $n$ extra individuals, uniparental pedigrees with $n+1$ generations are achieved. Thus, considering $n$ extra individuals, the same number of pedigrees can be considered as possibilities to 'construct' biparental pedigrees. Hence, considering biparental pedigrees, each pedigree associated with two uniparental pedigrees with different numbers $n_{1}$ and $n_{2}$ of extra individuals, respectively, $n_{1} \cdot n_{2}$ biparental pedigrees can be constructed as belonging to the same autosomal kinship class. However, considering classes of biparental pedigrees where both associated uniparental ped- igrees have the same number $n_{1}$ of extra individuals, symmetrical situations have to be discarded. In this case, it has been proved (see Appendix IV) that there are $\left(n^{2}{ }_{1}+n_{1}\right) / 2$ biparental pedigrees belonging to that class. In table 8 , the number of pedigrees belonging to each biparental class is presented considering the general case and the case of the autosomal kinship classes presented in table 7. Two biparental pedigrees belong to the same autosomal kinship class if and only if they have associated uniparental pedigrees, each one with the same number of extra individuals.

Finally, considering a total number $n$ of extra individuals, $n / 2$ (if $n$ is even) or $(n-1) / 2$ (if $n$ is odd) autosomal 
Table 8. Number of biparental pedigrees (BP) belonging to each biparental autosomal kinship class considering the general case and the case of the autosomal kinship classes presented in table 7

\begin{tabular}{|c|c|c|}
\hline \multicolumn{2}{|c|}{ Biparental pedigrees: $\mathrm{BP}=\mathrm{P}_{1}+\mathrm{P}_{2}$} & \multirow{2}{*}{$\begin{array}{l}\text { No. of biparental pedigrees } \\
\text { belonging to the same } \\
\text { autosomal kinship class }\end{array}$} \\
\hline $\begin{array}{l}\text { No. of extra indivi- } \\
\text { duals associated } \\
\text { with uniparental } \\
\text { pedigree } P_{1}\end{array}$ & $\begin{array}{l}\text { No. of extra indi- } \\
\text { viduals associated } \\
\text { with uniparental } \\
\text { pedigree } \mathrm{P}_{2}\end{array}$ & \\
\hline 1 & 2 & 2 \\
\hline 2 & 2 & 3 \\
\hline 1 & 3 & 3 \\
\hline$\ldots$ & $\ldots$ & $\ldots$ \\
\hline$n_{1}$ & $n_{2}$ & $\begin{array}{ll}n_{1} \cdot n_{2} & \text { if } n_{1} \neq n_{2} \\
\left(n_{1}^{2}+n_{1}\right) / 2 & \text { if } n_{1}=n_{2}\end{array}$ \\
\hline
\end{tabular}

Remember that two biparental pedigrees belong to the same autosomal kinship class if and only if they have associated uniparental pedigrees, each one with the same number of extra individuals.

kinship classes of biparental pedigrees involving two non-inbred individuals can be achieved. For a mathematical proof, see Appendix V.

\section{Conclusions}

The study of genetic relationships involving extended pedigrees has been a matter of great theoretical and practical interest, not only in humans but also in animal and plant studies, specifically for breeding applications. As a basis for this research on kinships we considered the root concept of IBD which is crucial to the study of relationships because the algebraic expressions for joint genotypic probabilities only depend on the IBD probabilities and on allele frequencies.

It is well known that some pedigrees have the same IBD partitions and are therefore indistinguishable using unlinked autosomal markers; some examples are circumstantially given in the literature. The example of the set of pedigrees avuncular, grandparent-grandchild and half-sibling, involving two non-inbred individuals, is most frequently referred to. See, for example, the paper by Egeland and Sheehan [10], where the particular case of this set is addressed to promote the use of linked autosomal markers in forensics because for those markers, the pedigrees referred to have different algebraic expressions. In this paper, discussing unlinked autosomal markers, we develop a theoretical framework that supports the de-
Table 9. Complete sets of pedigrees involving two non-inbred individuals belonging to the autosomal kinship classes referred in the most complete table we found in literature [table 2 in 8 ]

\begin{tabular}{|c|c|c|c|}
\hline \multirow[t]{2}{*}{ Pedigrees } & \multicolumn{3}{|c|}{ IBD partitions } \\
\hline & $k_{0}$ & $k_{1}$ & $k_{2}$ \\
\hline Unrelated $(*)$ & 1 & 0 & 0 \\
\hline $\begin{array}{l}\text { Identity }(*) \\
\text { Identical twins }\left(^{*}\right)\end{array}$ & 0 & 0 & 1 \\
\hline $\begin{array}{l}\text { Parent-child }\left({ }^{*}\right) \\
\text { Full-siblings }\left({ }^{*}\right)\end{array}$ & $\begin{array}{l}0 \\
1 / 4\end{array}$ & $\begin{array}{l}1 \\
1 / 2\end{array}$ & $\begin{array}{l}0 \\
1 / 4\end{array}$ \\
\hline $\begin{array}{l}\text { Avuncular }\left(^{*}\right) \\
\text { Half-siblings }\left(^{*}\right) \\
\text { Grandparent-grandchild }\left(^{*}\right)\end{array}$ & $1 / 2$ & $1 / 2$ & 0 \\
\hline $\begin{array}{l}\text { First cousins }\left({ }^{*}\right) \\
\text { Half-avuncular }\left(^{*}\right) \\
\text { Great-grand parent - great-grand child } \\
\text { Grand avuncular }\end{array}$ & $3 / 4$ & $1 / 4$ & 0 \\
\hline $\begin{array}{l}\text { Double first cousins }\left(^{*}\right) \\
\text { Double half-avuncular } \\
\text { First cousins whose non full-siblings } \\
\quad \text { parents are parent-child }\end{array}$ & $9 / 16$ & $6 / 16$ & $1 / 16$ \\
\hline $\begin{array}{l}\text { Half-siblings whose non-identical } \\
\text { parents are full-siblings }\left({ }^{*}\right) \\
\text { Half-siblings whose non-identical } \\
\text { parents are parent-child }\left(^{*}\right)\end{array}$ & $3 / 8$ & $1 / 2$ & $1 / 8$ \\
\hline $\begin{array}{l}\text { Half-siblings whose non-identical } \\
\text { parents are half-siblings }\left(^{*}\right) \\
\text { Half-siblings whose non-identical } \\
\text { parents are grandparent-grandchild } \\
\text { Half-siblings whose non-identical } \\
\text { parents are avuncular }\end{array}$ & $7 / 16$ & $1 / 2$ & $1 / 16$ \\
\hline
\end{tabular}

Pedigrees previously referred to in the mentioned table are marked with $\left(^{*}\right)$.

scription of pedigrees with the same IBD partitions, along with the achievement of those probabilities, based on a simple counting rule.

Concerning the kinship classes in the most complete table of IBD probabilities of some pedigrees involving two non-inbred individuals that we found in literature [table 2 in 8], we present all the genealogical relationships belonging to those classes in table 9, regardless of their frequency in the population. As mentioned previously, it must be stressed that to construct biparental pedigrees the uniparental pedigrees where both parents of one individual are ancestors of the other by the same path must be discarded because we are excluding inbreeding. 
With regard to uniparental pedigrees, it has been demonstrated that two pedigrees belong to the same autosomal kinship class if and only if they have the same number of extra individuals. Moreover, the number of different pedigrees belonging to each autosomal kinship class also depends only on the number of extra branches considered.

On the other hand, two biparental pedigrees belong to the same autosomal kinship class if and only if they can be decomposed into two uniparental pedigrees with, respectively, the same number of extra individuals. Indeed, the IBD partitions, as well as the number of possible pedigrees belonging to each class, do not depend on the total number of extra individuals considered but on the number of extra branches involved, respectively, in each component uniparental pedigree.

\section{Acknowledgements}

The authors would like to thank Leonor Gusmão (IPATIMUP) for very helpful comments on the manuscript. This work was partially supported by the Fundação para a Ciência e a Tecnologia through POCI 2010 (Programa Operacional Ciência e Inovação 2010) and PhD grant SFRH/BD/37261/2007. IPATIMUP is an Associate Laboratory of the Portuguese Ministry of Science, Technology and Higher Education and is partially supported by FCT.

\section{Appendix I}

Considering a uniparental pedigree for which it is necessary to consider $\mathrm{n}$ extra branches

$$
k_{0}^{(n)}=1-(1 / 2)^{n}, k_{1}^{(n)}=(1 / 2)^{n}, \mathrm{k}_{2}^{(n)}=0,
$$

where $k_{i}^{(n)}, i=0,1,2$, is the $i$-th IBD partition associated with the pedigree.

\section{Proof:}

The sentence can be proved by mathematical induction.

For $n=1: k_{0}^{(1)}=k_{1}^{(1)}=1 / 2$ and $k_{2}^{(1)}=0$, which are the IBD partitions associated with the obtained pedigrees when considering one extra branch: half-siblings, grandparent-grandchild and avuncular.

$$
\begin{aligned}
& \text { Suppose: } \\
& k_{0}^{(n)}=1-(1 / 2)^{n}, k_{1}^{(n)}=(1 / 2)^{n} \text {, and } \mathrm{k}_{2}^{(n)}=0 .
\end{aligned}
$$

For $n+1: k_{0}^{(n+1)}=k_{0}^{(n)}+1 / 2 k_{1}^{(n)}$. To obtain this expression consider the 'previous' pedigree with $n$ extra branches, where one extra individual has to be linked to a 'previously' extreme individual in order to obtain a pedigree with $n+1$ extra individuals. Then there are two possibilities for the extreme (and available) individuals in the pedigree with $n+1$ extra branches not sharing any IBD allele, either: the available individuals in the 'previous' pedigree (with $n$ extra branches) do not share any IBD allele: $k_{0}^{(n)}$, or they share one IBD allele and the individual where the new extra branch was linked transmitted the allele that is not IBD: $1 / 2 k_{1}^{(n)}$.

$$
\begin{aligned}
& k_{0}^{(n+1)}=k_{0}^{(n)}+\frac{1}{2} k_{1}^{(n)}= \\
& 1-\left(\frac{1}{2}\right)^{n}+\frac{1}{2} \cdot\left(\frac{1}{2}\right)^{n}=\sum_{i=1}^{n}\left(\frac{1}{2}\right)^{i}+\left(\frac{1}{2}\right)^{n+1}=\sum_{i=1}^{n+1}\left(\frac{1}{2}\right)^{i}=1-\left(\frac{1}{2}\right)^{n+1}
\end{aligned}
$$

On the other hand, $k_{1}^{(n+1)}=k_{2}^{(n)}+1 / 2 k_{1}^{(n)}$. With the same arguments as before consider the 'previous' pedigree with $n$ extra individuals and the extra branch that has to be linked to a 'previously' extreme individual to obtain a pedigree with $n+1$ extra branches. In this case, we have to consider the possibility of the available individuals in the 'previous' pedigree sharing two IBD alleles: $k_{2}^{(n)}$ (in such a case the individual where the new branch was attached always transmits one IBD allele concerning the alleles of the remaining extreme individual, the one that was available in the pedigree with $n$ extra individuals and that remains available when considering the pedigree with $n+1$ extra branches), and the possibility of the extreme individuals considered in the 'previous' pedigree with $n$ extra individuals sharing one IBD allele $k_{1}^{(n)}$ and the individual where the extra branch was linked transmitting the IBD allele $1 / 2 k_{1}^{(n)}$.

$$
\text { Thus, } k_{1}^{(n+1)}=k_{2}^{(n)}+1 / 2 k_{1}^{(n)}=1 / 2 \cdot(1 / 2)^{n}=(1 / 2)^{n+1} .
$$

Finally, $k_{2}^{(n+1)}=0$, because when one extra individual is added to a pedigree, at least the allele he or she received from the unrelated path is not IBD when compared with the extreme individual that remained from the 'previous' pedigree with n extra branches.

\section{Appendix II}

Considering the autosomal kinship class of uniparental pedigrees with $n$ extra individuals, there are $n+2$ different pedigrees belonging to that class.

Proof:

To prove this sentence we consider separately the pedigrees achieved when the extra individuals are added to the atom pedigree full-siblings and those achieved when the atom pedigree considered is the pedigree parent-child.

Considering the atom pedigree full-siblings and $\mathrm{n}$ extra branches we can distribute them $n+1$ different ways: $(0, n),(1$, $n-1),(2, n-2), \ldots,(n-1,1),(n, 0)$, where the first and second numbers in each ordered pair correspond to the extra individuals successively linked to the full-sibling to the left and right side, respectively. However, pedigrees obtained when considering $(i, n-$ $i)$ and $(n-i, i)$ extra individuals are symmetric and equivalent. Thus, considering $\mathrm{n}$ extra individuals and the atom pedigree fullsiblings we obtain $(n / 2)+1$ (if $n$ is even) or $(n-1) / 2+1$ (if $n$ is odd) different pedigrees.

On the other hand, considering $\mathrm{n}$ extra individuals and the atom pedigree parent-child, there are $n+1$ different ways of linking the $\mathrm{n}$ extra branches. Indeed, for all possible values, if $i$ individuals are successively linked at the 'parent', $n-i$ individuals 
have to be successively linked at the 'child'. In the end, we just have to see how many different ways we can distribute $n+1$ individuals at the right and left side of the 'central' individual 'parent' at the atom pedigree parent-child, considering that at least one individual (the 'child') has to remain at the right side.

With analogous notation as above consider $(i, n-i)$ the pedigree achieved when considering $i$ extra individuals successively linked to the parent and $n-i$ linked to the child. This corresponds to the pedigree when i individuals are linked at the left side of the 'parent' at the atom pedigree parent-child and $n-i+1$ at the right side of that individual. Thus, considering that the pedigrees achieved with $(i, n-i+1)$ successively linked individuals at the left and right side of the parent are equivalent and symmetric to those when considering $(n-i+1, i-1)$ extra individuals, there are $n / 2+1$ (if $n$ is even) and $(n-1) / 2+1$ (if $n$ is odd) different pedigrees that can be depicted considering the atom pedigree parentchild.

Thus, considering the number $n$ of extra individuals:

\begin{tabular}{llll}
\hline & \multicolumn{2}{l}{ Atom pedigrees considered } & \multirow{2}{*}{ Total } \\
\cline { 2 - 3 } & full-siblings & parent-child & \\
\hline$n$ even & $n / 2+1$ & $n / 2+1$ & $n+2$ \\
$n$ odd & $(n-1) / 2+1$ & $(n+1) / 2+1$ & $n+2$ \\
\hline
\end{tabular}

\section{Appendix III}

Two biparental pedigrees belong to the same autosomal kinship class if and only if the associated uniparental pedigrees have the same number of extra individuals.

Proof:

By definition, two pedigrees belong to the same autosomal kinship class if and only if they have the same IBD partitions.

For biparental pedigrees, considering that $n_{1}$ and $n_{2}$ are the extra individuals associated with the uniparental pedigrees:

$$
\begin{aligned}
& k_{0}=1-(1 / 2)^{n_{1}}-(1 / 2)^{n_{2}}+(1 / 2)^{n_{1}+n_{2}}, \\
& k_{1}=(1 / 2)^{n_{1}}+(1 / 2)^{n_{2}}-(1 / 2)^{n_{1}+n_{2}-1} \text { and } k_{2}=(1 / 2)^{n_{1}+n_{2}} .
\end{aligned}
$$

Consider two biparental pedigrees: $\mathrm{BP}_{1}$ and $\mathrm{BP}_{2}$, composed by two pairs of uniparental pedigrees with $\left(n_{1}, n_{2}\right)$ and $\left(n_{3}, n_{4}\right)$ extra associated individuals, respectively.

$(<=)$ Of course, if that $\left(n_{1}=n_{3}\right.$ and $\left.n_{2}=n_{4}\right)$ or $\left(n_{1}=n_{4}\right.$ and $\left.n_{2}=n_{3}\right)$ then $k_{0}\left(\mathrm{BP}_{1}\right)=k_{0}\left(\mathrm{BP}_{2}\right)$ and $k_{1}\left(\mathrm{BP}_{1}\right)=k_{1}\left(\mathrm{BP}_{2}\right)$ and $k_{2}\left(\mathrm{BP}_{1}\right)=k_{2}\left(\mathrm{BP}_{2}\right)$, and the biparental pedigrees belong to the same autosomal kinship class.

$(=>)$ Consider that biparental pedigrees $\mathrm{BP}_{1}$ and $\mathrm{BP}_{2}$ belong to the same autosomal kinship class.

Then,

$$
\begin{aligned}
k_{0}\left(B P_{1}\right) & =k_{0}\left(B P_{2}\right) \Leftrightarrow 1-(1 / 2)^{n_{1}}-(1 / 2)^{n_{2}}+(1 / 2)^{n_{1}+n_{2}} \\
& =1-(1 / 2)^{n_{3}}-(1 / 2)^{n_{4}}+(1 / 2)^{n_{3}+n_{4}}, \\
k_{1}\left(B P_{1}\right) & =k_{1}\left(B P_{2}\right) \Leftrightarrow(1 / 2)^{n_{1}}+(1 / 2)^{n_{2}}-(1 / 2)^{n_{1}+n_{2}} \\
& =(1 / 2)^{n_{3}}+(1 / 2)^{n_{4}}-(1 / 2)^{n_{3}+n_{4}} \text { and } \\
k_{2}\left(B P_{1}\right) & =k_{2}\left(B P_{2}\right) \Leftrightarrow(1 / 2)^{n_{1}+n_{2}}=(1 / 2)^{n_{3}+n_{4}} .
\end{aligned}
$$

These three sentences can be condensed into two:

$$
(1 / 2)^{n_{1}}+(1 / 2)^{n_{2}}=(1 / 2)^{n_{3}}+(1 / 2)^{n_{4}}
$$

and

$$
n_{1}+n_{2}=n_{3}+n_{4}
$$

Consider $n_{1} \leq n_{2}$. If $n_{1}<n_{3}$ and $n_{1}<n_{4}$ then

$$
\begin{aligned}
(1 / 2)^{n_{3}}+(1 / 2)^{n_{4}} & \leq 2 \max \left[(1 / 2)^{n_{3}},(1 / 2)^{n_{4}}\right] \\
& \leq(1 / 2)^{n_{1}}<(1 / 2)^{n_{1}}+(1 / 2)^{n_{2}} .
\end{aligned}
$$

Thus, by (1) we can infer that: $n_{1} \geq \min \left\{n_{3}, n_{4}\right\}$. The same argument implies, by symmetry, that: $\min \left\{n_{3}, n_{4}\right\} \geq n_{1}$. Hence, $n_{1}=$ $n_{3}$ or $n_{1}=n_{4}$. By (2), it follows that: $\left(n_{1}=n_{3}\right.$ and $\left.n_{2}=n_{4}\right)$ or $\left(n_{1}=\right.$ $n_{4}$ and $\left.n_{2}=n_{3}\right)$.

\section{Appendix IV}

Considering biparental pedigrees where the associated uniparental pedigrees have the same number $\mathrm{n}$ of extra individuals, there are $\left(n^{2}+n\right) / 2$ different biparental pedigrees.

Proof:

It is known that considering $n$ extra individuals, there are $n$ uniparental pedigrees that can be used to 'construct' biparental pedigrees because we are just considering non-inbred individuals (see text). Thus, we can consider $n$ possibilities for achieving biparental pedigrees where both uniparental pedigrees associated are the same. Moreover, we can choose $C_{2}^{n}$, combinations of two from a set with $n$ pedigrees, considering that the uniparental pedigrees associated are different.

Thus,

$$
n+C_{2}^{n}=n+n ! /[2(n-2) !]=n+[n(n-1)] / 2=\left(n^{2}+n\right) / 2 .
$$

\section{Appendix V}

Considering $n$ extra individuals, there are $n / 2$ (if $n$ is even) or $(n-1) / 2$ (if $n$ is odd) autosomal kinship classes of biparental pedigrees.

\section{Proof:}

It is known that different bipartitions of $n$ (corresponding to the number of extra individuals in each uniparental pedigree associated with the biparental pedigree) achieve different classes of biparental pedigrees (see text and Appendix III). Thus, considering $n$ extra individuals there are $n$ bipartitions of that same number because at least one extra individual has to be considered in each associated uniparental pedigree. Therefore, and considering that the achieved biparental pedigrees with the bipartitions of $n$, namely $\left(n_{1}, n_{2}\right)$ and $\left(n_{2}, n_{1}\right)$, are equivalent, there are $n / 2$ (if $n$ is even) or $(n-1) / 2$ (if $n$ is odd) autosomal kinship classes of biparental pedigrees considering $n$ extra individuals. 


\section{References}

1 Jobling M, Gill P: Encoded evidence: DNA in forensic analysis. Nat Rev Genet 2004;5:739752.

-2 Karlsson A, Holmlund G, Egeland T, Mostad P: DNA-testing for immigration cases: The risk of erroneous conclusions. Forensic Sci Int 2007;172:144-149.

$\checkmark 3$ Dawid A, Mortera J, Pascali V, Boxel D: Probabilistic Expert Systems for forensic inference from genetic markers. Scand J Statistic 2002;29:577-595.

$\checkmark 4$ Brenner C: Some mathematical problems in the DNA identification of victims in the 2004 tsunami and similar mass fatalities. Forensic Sci Int 2006;157:172-180.

$\checkmark 5$ Weir B, Anderson A, Hepler A: Genetic relatedness analysis: modern data and new challenges. Nat Rev Genet 2006;7:771-780.

-6 Gasbarra D, Pirinen M, Sillanpaa, Salmela E, Arjas E: Estimating genealogies from unlinked marker data: a Bayesian approach. Theor Popul Biol 2007;72:305-322.
Blouin M: DNA-based methods for pedigree reconstruction and kinship analysis in natural populations. Trends Ecol Evol 2003;18: 503-511.

8 Thompson E: The estimation of pairwise relationships. Ann Hum Genet 1975;39:173188.

9 Thompson E, Meagher T: Genetic linkage in the estimation of pairwise relationships. Theor Appl Genet 1998;97:857-864.

10 Egeland T, Sheehan N: On identification problems requiring linked autosomal markers. Forensic Sci Int Genet 2008;2:219-225.

11 Pinto N, Gusmão L, Amorim A: X-chromosome markers in kinship testing: a generalisation of the IBD approach identifying situations where their contribution is crucial. Forensic Sci Int Genet 2010, Doi: 10.1016/j. fsigen.2010.01.011.
12 Jacquard A: The genetic structure of populations; in Biomathematics. Springer-Verlag, Berlin, Heidelberg, New York, 1974, vol 5, pp 102-107.

13 Pinto N, Gusmão L, Amorim A: Likelihood ratios in kinship analysis: contrasting kinship classes, not genealogies. Forensic Sci Int Genet 2010;4:218-219.

14 Donnelly K: The probability that related individuals share some section of genome identical by descent. Theor Popul Biol 1983; 23:34-63.

15 Skare O, Sheehan N, Egeland T: Identification of distant family relationships. Bioinformatics 2009;25:2376-2382.

16 Gill P, Kimpton C, Aliston-Greiner R, Sullivan K: Establishing the identity of Anna Anderson Manahan. Nat Genet 1995;9:9-10.

17 Nothnagel M, Schmidtke J, Krawczak M: Potentials and limits of pairwise kinship analysis using autosomal short tandem repeat loci. Int J Legal Med 2010;124:205-215 